Research in Astron. Astrophys. 2015 Vol. X No. XX, 000-000

http://www.raa-journal.org http://www.iop.org/journals/raa

$\boldsymbol{R}$ esearch in

Astronomy and

Astrophysics

\title{
Discovery of a strongly $r$-process enhanced extremely metal-poor star LAMOST J110901.22+075441.8
}

\author{
Haining $\mathrm{Li}^{1}$, Wako Aoki ${ }^{2,3}$, Satoshi Honda ${ }^{4}$, Gang Zhao ${ }^{1}$, Norbert Christlieb ${ }^{5}$, Takuma Suda ${ }^{6}$ \\ 1 Key Lab of Optical Astronomy, National Astronomical Observatories, Chinese Academy of \\ Sciences, A20 Datun Road, Chaoyang, Beijing 100012, China; Ihn@nao.cas.cn \\ 2 National Astronomical Observatory of Japan, 2-21-1 Osawa, Mitaka, Tokyo, 181-8588, Japan \\ 3 Department of Astronomical Science, School of Physical Sciences, The Graduate University of \\ Advanced Studies (SOKENDAI), 2-21-1 Osawa, Mitaka, Tokyo 181-8588, Japan \\ 4 University of Hyogo, 407-2, Nishigaichi, Sayo-cho, Sayo, Hyogo, 679-5313, Japan \\ 5 Zentrum für Astronomie der Universität Heidelberg, Landessternwarte, Königstuhl 12, D-69117 \\ Heidelberg, Germany \\ ${ }^{6}$ Research Center for the Early Universe, The University of Tokyo, Hongo 7-3-1, Bunkyo-ku, Tokyo \\ 113-0033, Japan
}

Received 2015 April 02; accepted 2015 March 13

\begin{abstract}
We report the discovery of an extremely metal-poor (EMP) giant, LAMOST J110901.22+075441.8, which exhibits large excess of $r$-process elements with $[\mathrm{Eu} / \mathrm{Fe}] \sim+1.16$. The star is one of the newly discovered EMP stars identified from LAMOST low-resolution spectroscopic survey and the high-resolution followup observation with the Subaru Telescope. Stellar parameters and elemental abundances have been determined from the Subaru spectrum. Accurate abundances for a total of 23 elements including 11 neutron-capture elements from $\mathrm{Sr}$ through Dy have been derived for LAMOST J110901.22+075441.8. The abundance pattern of LAMOST J110901.22+075441.8 in the range of $\mathrm{C}$ through $\mathrm{Zn}$ is in line with the "normal" population of EMP halo stars, except that it shows a notable underabundance in carbon. The heavy element abundance pattern of LAMOST J110901.22+075441.8 is in agreement with other well studied cool $r$-II metal-poor giants such as CS 22892-052 and CS 31082-001. The abundances of elements in the range from Ba through Dy well match the scaled Solar $r$-process pattern. LAMOST J110901.22+075441.8 provides the first detailed measurements of neutron-capture elements among $r$-II stars at such low metallicity with $[\mathrm{Fe} / \mathrm{H}] \lesssim-3.4$, and exhibits similar behavior in the abundance ratio of $[\mathrm{Zr} / \mathrm{Eu}]$ as well as $\mathrm{Sr} / \mathrm{Eu}$ and $\mathrm{Ba} / \mathrm{Eu}$ as other $r-$ II stars.
\end{abstract}

Key words: star: abundances — stars: Population II — nucleosynthesis

\section{INTRODUCTION}

Detailed elemental abundances of metal-poor stars in the Galactic halo provide fundamental knowledge about the history and nature of the nucleosynthesis in the Galaxy. Particularly, abundances of the slow $(s)$ and rapid $(r)$ neutron-capture elements are of great importance to constrain early Galactic nucleosynthesis and chemical evolution. In the past decades, great efforts have been devoted to studies in relevant fields based on high-resolution spectroscopic measurements of the elemental abundances of 
metal-poor stars in the Galaxy. These observations together with theoretical studies have revealed that the $r$-process is primarily responsible for the production of heavy elements beyond the iron group (Spite \& Spite 1978; Sneden et al. 1996) in the early Galaxy. Only at later time (higher metallicities), the onset of the $s$-process occurs, injecting nucleosynthesis material into the interstellar medium from long-lived low- and intermediate mass stars (Burris et al. 2000). Detections of radioactive elements including thorium and uranium in a few $r$-process enhanced metal-poor stars have provided an independent measurement on the age of these oldest stars, and hence set lower limits to the age of the Galaxy (Hill et al. 2002; Frebel et al. 2007).

Sneden et al. (1994) found the first $r$-process enhanced extremely metal-poor (EMP) giant CS 22892-052, with a [Eu/Fe] $\sim+1.6^{1}$ and an abundance pattern from Ba through Dy which is similar to a scaled Solar System $r$-process (SSr) distribution. A few rare stars have subsequently been found to exhibit extreme enhancements in $r$-process elements, suggesting that the observed abundances are dominated by the effect from a single or very few nucleosynthesis events. Based on the definition by Beers \& Christlieb (2005), such stars with $[\mathrm{Eu} / \mathrm{Fe}]>+1$ and $[\mathrm{Ba} / \mathrm{Eu}]<0$ are referred to $r-$ II stars. Although the astrophysical site of the $r$-process is not yet clear, the $r$-process is believed to be connected to explosive conditions of massive-star core-collapse supernovae (Woosley et al. 1994), or neutron star mergers (Goriely et al. 2013). Therefore, $r$-II stars are the best candidates to explore the details of the $r$-process and its site.

There are 12 r-II stars known to date (e.g., Hill et al. 2002; Sneden et al. 2003; Honda et al. 2004; Sneden et al. 2008; Hayek et al. 2009; Mashonkina et al. 2010; Aoki et al. 2010). Detailed abundance analysis of these $r$-II stars have found excellent match between the stellar and solar $r$-process pattern in the Ba-Hf range, which indicates a universal $r$-process, i.e, elements were produced with the same proportions during the evolution of the Galaxy. Such conclusion is of fundamental importance for a better understanding of the nature of the $r$-process. To establish the origin of the heavy elements beyond the iron group among the oldest stars in the Galaxy, larger samples of accurate measurements of additional elements are required.

LAMOST J110901.22+075441.8 was identified as a candidate of EMP stars from the first data release (Luo et al. 2015) of the low-resolution $(R \sim 1800)$ spectroscopic survey of LAMOST ${ }^{2}$ (the Large sky Area Multi-Object fiber Spectroscopic Telescope, also known as Wang-Su Reflecting Schmidt Telescope or Guoshoujing Telescope; Zhao et al. 2006; Cui et al. 2012; Zhao et al. 2012; Luo et al. 2012). The follow-up high-resolution spectroscopic observation was carried out with the Subaru Telescope (Li et al. 2015b), which has confirmed its being a $r$-II EMP giant. In this paper, we introduce the observation and measurements of parameters and abundances of LAMOST J110901.22+075441.8 in Section 2; results and interpretations on the elemental abundance are presented in Section 3; patterns of the heavy elements and conclusions are described in Sections 4 and 5.

\section{OBSERVATIONS AND MEASUREMENTS}

\subsection{Target selection and follow-up observations}

LAMOST J110901.22+075441.8 was selected as a candidate of EMP stars from the low-resolution spectra of the first data release of the LAMOST spectroscopic survey. The wavelength coverage (3700$9100 \AA)$ and resolving power $(R=1800)$ of the LAMOST spectra (Fig. 1) allow a robust estimation of the stellar parameters including metallicities. Methods to determine the metallicity of an object and the selection of EMP candidates were similar to those made by Li et al. (2015).

For 54 candidates of extremely metal-poor stars selected from the LAMOST sample,"snapshot" high-resolution spectra were acquired with the resolving power $R=36,000$ and exposure times of 10-20 minutes during the two-night run in May 2014 with Subaru/HDS as made by Aoki et al. (2013). LAMOST J110901.22+075441.8 was observed on May 9. Since this object is relatively bright $(r=$

\footnotetext{
$1[A / B]=\log \left(N_{A} / N_{B}\right)_{\star}-\log \left(N_{A} / N_{B}\right)_{\odot}$, where $N_{A}$ and $N_{B}$ are the number densities of elements $\mathrm{A}$ and $\mathrm{B}$ respectively, and $\star$ and $\odot$ refer to the star and the Sun respectively

2 See http://www.lamost.org for more detailed information, and the progress of the LAMOST surveys.
} 


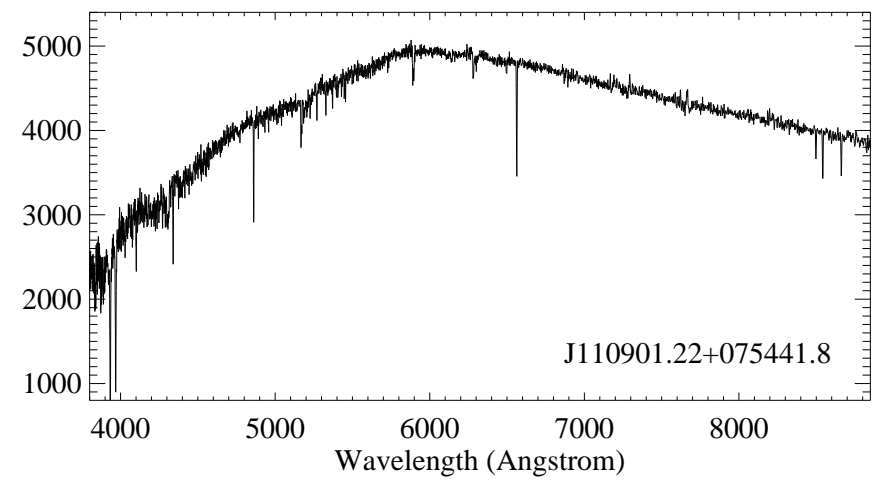

Fig. 1 Medium-resolution spectrum of the r-II star obtained by LAMOST.

12.08), quite high signal-to-noise ratio was achieved covering $4000-6800 \AA$, e.g., with a $S / \mathrm{N}$ of about 70 at $4500 \AA$ and 110 at $5200 \AA$ by an exposure of 10 minutes. Data reduction was carried out with standard procedures using the IRAF echelle package including bias-level correction, scattered light subtraction, flat-fielding, extraction of spectra, and wavelength calibration using $\mathrm{Th}-\mathrm{Ar}$ arc lines. Cosmic-ray hits were removed by the method described in Aoki et al. (2005).

Radial velocities of the sample were obtained using the IRAF procedure fxcor, and a synthetic spectrum with low-metallicity was employed as a template for cross-correlation. The above method derives a radial velocity of $-72.3 \pm 0.4 \mathrm{~km} \mathrm{~s}^{-1}$.

\subsection{Stellar parameters}

Equivalent widths were measured by fitting Gaussian profiles to isolated atomic absorption lines based on the line list of Aoki et al. (2013) for elements lighter than Zn, and that from Mashonkina et al. (2010) for heavy elements beyond $\mathrm{Sr}$.

Since there is not yet a uniform photometric system for all the LAMOST input catalogue, we have adopted the spectroscopic method to derive the stellar parameters of the whole sample, including LAMOST J110901.22+075441.8. By minimizing the trend of the relationship between the derived abundances and excitation potentials of Fe I lines, the effective temperature $T_{\text {eff }}$ of LAMOST J110901.22+075441.8 was determined. The empirical formula derived by Frebel et al. (2013) has been adopted to correct the usually expected systematic offsets between the spectroscopic and photometric effective temperatures, which resulted in a $T_{\text {eff }}$ of $4441 \mathrm{~K}$ from the original value of $4190 \mathrm{~K}$. The microturbulent velocity $\xi$ was also determined based on analysis of Fe I lines, i.e., by forcing the iron abundances of individual lines to exhibit no dependence on the reduced equivalent widths. The sufficiently high quality of the Subaru spectrum allows us to detect $11 \mathrm{Fe}$ II lines for LAMOST J110901.22+075441.8. Therefore the surface gravity $\log g$ was determined by minimizing the difference between the average abundances derived from the Fe I and Fe II lines.

The derived parameters of LAMOST J110901.22+075441.8 are listed in Table 1. Since the $V-K$ color is available for LAMOST J110901.22+075441.8 $\left((V-K)_{0}=2.54\right)$, we have also checked the photometric temperature of this object. Adopting the calibration by Alonso et al. $(1999,2001)$, the derived temperature is $4560 \mathrm{~K}$, which is consistent with the spectroscopic temperature within the level of the uncertainty of our measurement. 


\subsection{Abundance determination}

For the abundance analysis, the 1D plane-parallel, hydrostatic model atmospheres of the ATLAS NEWODF grid of Castelli \& Kurucz (2003) were adopted, assuming a mixing-length parameter of $\alpha_{\mathrm{MLT}}=1.25$, no convective overshooting, and local thermodynamic equilibrium. An updated version of the abundance analysis code MOOG (Sneden 1973) was used, which does not treat continuous scattering as true absorption, but as a source function which sums both absorption and scattering (Sobeck et al. 2011). When calculating $[\mathrm{X} / \mathrm{H}]$ and $[\mathrm{X} / \mathrm{Fe}]$ abundance ratios, the photospheric Solar abundances of Asplund et al. (2009) were adopted .

Abundances of most elements were computed using the measured equivalent widths of isolated atomic lines with the derived stellar parameters. For heavy elements whose spectral lines show hyperfine splitting, such as Sr, Ba, La and Eu, the elemental abundances were determined using spectral synthesis taking into account the isotopic splitting and/or hyperfine structure (HFS). The derived abundances of LAMOST J110901.22+075441.8 are listed in Table 1, which also includes $N$, the number of lines which have been used to determine the abundance, together with the abundance error as described in the following text.

The uncertainties of the derived abundances mainly come from two aspects, i.e, the uncertainties of the equivalent width measurements, and those caused by the uncertainties of stellar parameters. In the case of equivalent width measurement, when $N \geq 2$ lines of individual species of an element were observed, the dispersion around the average abundance was used to present random error; if the elemental abundance was determined from a single line, the statistic error of the equivalent widths was estimated based on the classical formula of Cayrel (1988, Equation 7). The abundance uncertainties associated with the uncertainties of the stellar parameters were estimated by individually varying $T_{\text {eff }}$ by $+150 \mathrm{~K}, \log g$ by $+0.1 \mathrm{dex}$, and $\xi$ by $+0.1 \mathrm{~km} \mathrm{~s}^{-1}$ in the stellar atmospheric model. The total uncertainty of the errors have been computed as the quadratic sum of the above aspects, and are shown in the column of $\sigma$ in Table 1.

\section{ELEMENTAL ABUNDANCES AND INTERPRETATIONS}

In Fig. 2, abundance ratios relative to iron of LAMOST J110901.22+075441.8 are shown and compared to other metal-poor $r$-II stars, as well as the "normal" metal-poor giants from the "First Stars" project (Cayrel et al. 2004). Note that there is only one cool metal-poor, main-sequence $r$-II star discovered by Aoki et al. (2010) with $[\mathrm{Fe} / \mathrm{H}]=-3.36$, while the rest are all cool giants.

\subsection{Lithium through zinc}

Lithium. The Li I $6708 \AA$ line is covered in the observed spectrum, but we could not detect the lithium line in LAMOST J110901.22+075441.8. An upper limit of $A(\mathrm{Li})<0.35^{3}$ has been derived based on spectral synthesis. This is not unexpected for a red giant which is usually showing a rather low abundance of $\mathrm{Li}$, since such objects have already undergone the first dredge-up and the surface material is mixed with internal one with depleted lithium.

Carbon. The carbon abundance of LAMOST J110901.22+075441.8 was derived by matching the observed CH A-X band at $4310 \AA$ (i.e., the G-band) to the synthetic spectra. The object shows notable underabundant carbon compared to other $r$-II stars and most of the EMP stars (Fig. 2). The derived abundance ratio $[\mathrm{C} / \mathrm{Fe}]=-0.57$, which is close to the ratios of the so-called "mixed" EMP giants from the sample of Spite et al. (2005). Such low carbon abundance ratio is likely to be caused by mixing which has brought processed material to the surface from deep layers. Such cool giants should exhibit enhancement of nitrogen which is probably converted from carbon; however, due to limited wavelength coverage of the snapshot spectrum which does not include the $\mathrm{NH}$ or $\mathrm{CN}$ lines, we are unable to measure the nitrogen abundance of LAMOST J110901.22+075441.8 with current data.

\footnotetext{
$3 A(\mathrm{Li})$ is defined as $A(\mathrm{Li})=\log (N(\mathrm{Li}) / N(\mathrm{H}))+12$.
} 
Table 1 Stellar Parameters and Elemental abundances of LAMOST J110901.22+075441.8.

\begin{tabular}{|c|c|c|c|c|c|}
\hline & \multicolumn{4}{|c|}{ LAMOST J110901.22+075441.8 } & \multirow[t]{5}{*}{ Sun } \\
\hline$T_{\text {eff }}(\mathrm{K})$ & & $4440 \pm$ & & & \\
\hline $\log g$ & & $0.70 \pm$ & & & \\
\hline$[\mathrm{Fe} / \mathrm{H}]$ & & $-3.41=$ & & & \\
\hline$\xi\left(\mathrm{km} \mathrm{s}^{-1}\right)$ & & $1.98 \pm$ & & & \\
\hline Ton & $\log \epsilon(X)$ & [X/Fe] & $N$ & $\bar{\sigma}$ & $\log \epsilon(X)$ \\
\hline $\mathrm{C}$ & 4.45 & -0.57 & 1 & 0.38 & 8.43 \\
\hline $\mathrm{Na}$ & 3.41 & 0.58 & 2 & 0.23 & 6.24 \\
\hline $\mathrm{Mg}$ & 4.60 & 0.41 & 4 & 0.15 & 7.60 \\
\hline $\mathrm{Si}$ & 4.58 & 0.48 & 1 & 0.17 & 7.51 \\
\hline $\mathrm{Ca}$ & 3.30 & 0.31 & 15 & 0.16 & 6.34 \\
\hline $\mathrm{Sc}$ & -0.33 & -0.07 & 8 & 0.16 & 3.15 \\
\hline Ti I & 1.71 & 0.17 & 10 & 0.23 & 4.95 \\
\hline Ti II & 1.81 & 0.27 & 25 & 0.11 & 4.95 \\
\hline $\mathrm{Cr}$ & 1.83 & -0.40 & 5 & 0.20 & 5.64 \\
\hline $\mathrm{Fe} \mathrm{I}$ & 4.09 & 0.00 & 98 & 0.24 & 7.50 \\
\hline $\mathrm{Fe}$ II & 4.08 & -0.01 & 11 & 0.14 & 7.50 \\
\hline Co & 1.64 & 0.06 & 1 & 0.23 & 4.99 \\
\hline $\mathrm{Ni}$ & 2.75 & -0.06 & 2 & 0.16 & 6.22 \\
\hline $\mathrm{Zn}$ & 1.62 & 0.47 & 2 & 0.09 & 4.56 \\
\hline $\mathrm{Sr}$ & -0.25 & 0.29 & 2 & 0.19 & 2.87 \\
\hline Y & -1.20 & 0.00 & 3 & 0.12 & 2.21 \\
\hline $\mathrm{Zr}$ & -0.54 & 0.29 & 2 & 0.17 & 2.58 \\
\hline $\mathrm{Ba}$ & -0.92 & 0.31 & 3 & 0.14 & 2.18 \\
\hline $\mathrm{La}$ & -1.60 & 0.71 & 2 & 0.16 & 1.10 \\
\hline $\mathrm{Ce}$ & -1.47 & 0.36 & 2 & 0.13 & 1.58 \\
\hline $\operatorname{Pr}$ & -1.78 & 0.91 & 1 & 0.15 & 0.72 \\
\hline $\mathrm{Nd}$ & -1.47 & 0.52 & 4 & 0.16 & 1.42 \\
\hline $\mathrm{Sm}$ & -1.67 & 0.78 & 2 & 0.17 & 0.96 \\
\hline $\mathrm{Eu}$ & -1.73 & 1.16 & 1 & 0.14 & 0.52 \\
\hline Dy & -1.35 & 0.96 & 1 & 0.15 & 1.10 \\
\hline
\end{tabular}

Sodium. Abundance of Na was derived based on the EW measurements of the two resonance lines. It is noticed from Fig. 2 that the $[\mathrm{Na} / \mathrm{Fe}]=0.58$ of LAMOST J110901.22+075441.8 indicates relative excess compared to other $r-$ II stars which normally show solar or slight lower [Na/Fe] ratio. The difference may be balanced when the NLTE correction on sodium abundances are considered, e.g., according to Cayrel et al. (2004), a correction up to -0.5 dex shall be added to the LTE sodium abundance. However, we have only found $[\mathrm{Na} / \mathrm{Fe}]$ from literatures for a few $r$-II stars, and a larger sample would be needed to make any conclusive remark.

The $\alpha$-elements. For LAMOST J110901.22+075441.8, abundances of four $\alpha$-elements $\mathrm{Mg}, \mathrm{Si}$, $\mathrm{Ca}$, and $\mathrm{Ti}$ were derived based on the EW measurements of atomic lines. As shown in Fig. 2, all the $\alpha$-elements present enhancement relative to iron, with an average $[(\mathrm{Mg}+\mathrm{Si}+\mathrm{Ca}+\mathrm{Ti}) / \mathrm{Fe}]=+0.37$ which agrees with the canonical value of $[\alpha / \mathrm{Fe}] \sim+0.4$ for halo stars (McWilliam 1997).

Scandium and iron-peak elements. Except for $\mathrm{V}$ whose atomic lines are not covered and $\mathrm{Mn}$ for which only one line with distorted feature could be found, abundance of elements in the nuclear charge ranging from $Z=21$ through 28 were determined for LAMOST J110901.22+075441.8. The abundance ratio to iron of $\mathrm{Sc}, \mathrm{Co}$, and $\mathrm{Ni}$ are about the solar value. $\mathrm{Cr}$ is deficient relative to iron and solar ratios, with $[\mathrm{Cr} / \mathrm{Fe}]=-0.40$, while $\mathrm{Zn}$ is overabundant relative to iron with $[\mathrm{Zn} / \mathrm{Fe}]=+0.47$. These abundance ratios in LAMOST J110901.22+075441.8 well agree with the general trend of EMP halo stars with similar metallicities (with $\mathrm{Sc}, \mathrm{Cr}$, and $\mathrm{Zn}$ shown in Fig. 2 as examples).

In general, we found that the element abundance pattern of LAMOST J110901.22+075441.8 in the $\mathrm{C}-\mathrm{Zn}$ range resembles the "normal" pattern of halo EMP stars, except that it shows a quite low $\mathrm{C}$ abundance and relatively large $[\mathrm{Na} / \mathrm{Fe}]$. The underabundance in carbon is presumably due to the fact 


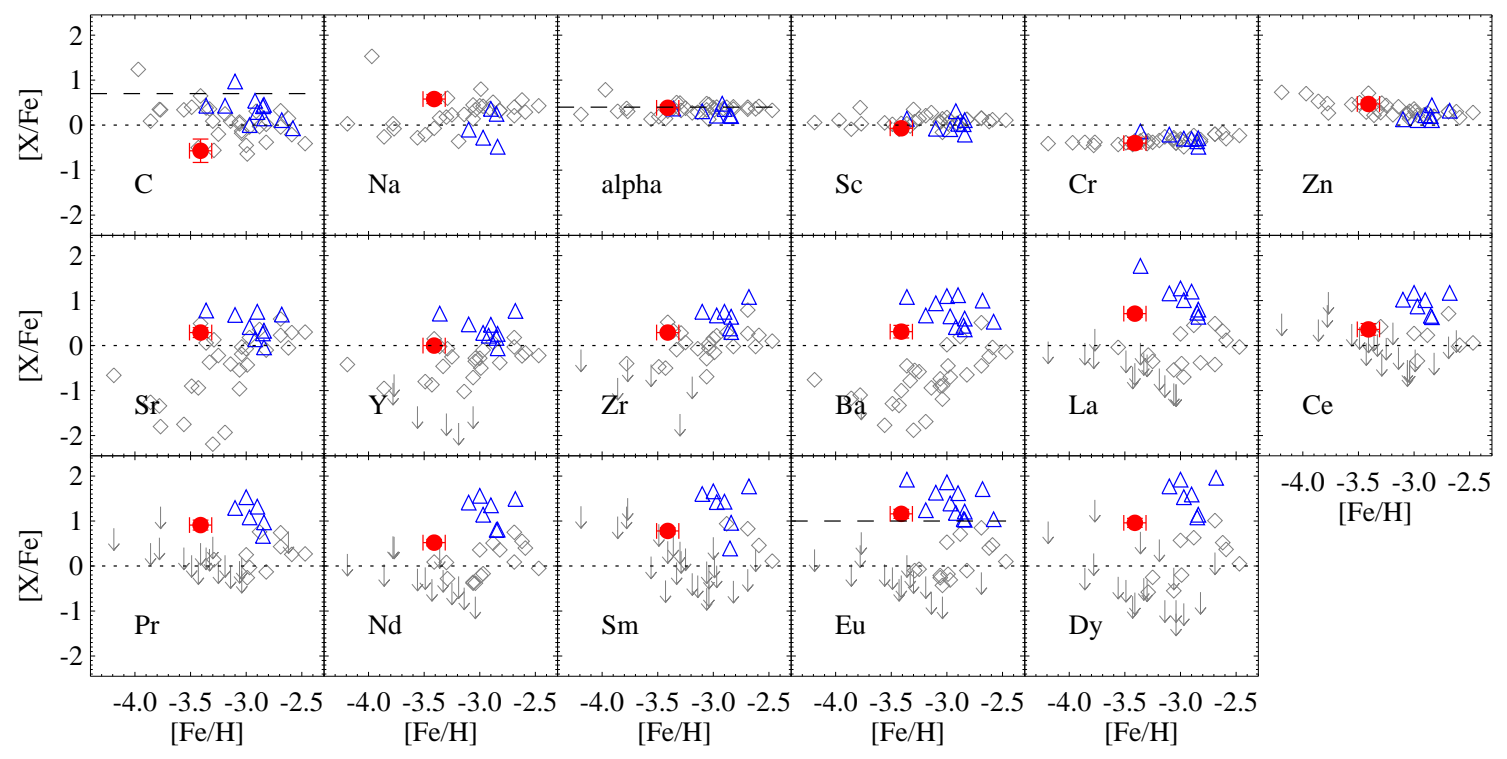

Fig. 2 [X/Fe] vs. [Fe/H] from C through Eu of LAMOST J110901.22+075441.8. Filled circles are abundances of LAMOST J110901.22+075441.8. Triangles are abundance ratios of other $r-$ II stars collected from literatures (Hill et al. 2002; Sneden et al. 2003; Honda et al. 2004; Christlieb et al. 2004; Barklem et al. 2005; François et al. 2007; Lai et al. 2008; Sneden et al. 2008; Hayek et al. 2009; Mashonkina et al. 2010; Aoki et al. 2010). Diamonds and downward arrows refer to abundances and upper-limits of metal-poor giants from the "First Stars" project (Cayrel et al. 2004; Spite et al. 2005; François et al. 2007). For C, the dashed line refers to the division between carbon-enhanced and carbon-normal of $[\mathrm{C} / \mathrm{Fe}] \sim+0.7$ (Aoki et al. 2007). For the $\alpha$-elements, the dashed line refers to the canonical value of $[\alpha / \mathrm{Fe}] \sim+0.4$ for halo stars (McWilliam 1997). For Eu, the criterion for $r-$ II stars with $[\mathrm{Eu} / \mathrm{Fe}] \geq+1.0$ (Beers \& Christlieb 2005) is also presented with a dashed line for reference.

that it is a more evolved star compared with EMP stars with higher temperatures. The overabundance in sodium may be explained by the discrepancy between LTE and NLTE Na abundances for EMP giants.

\subsection{Heavy element: strontium through dysprosium}

We determined abundances of LAMOST J110901.22+075441.8 for 11 heavy elements, including three light trans-iron elements and eight elements in the region of the second $r$-process peak. The elements representing the third peak could not be measured, because the wavelength coverage of our spectrum did not include any strong lines for measurements.

The light trans-iron elements. Three elements were measured in the region of the first peak with $38 \leq Z \leq 46$, including $\mathrm{Sr}, \mathrm{Y}$, and $\mathrm{Zr}$. The abundance of $\mathrm{Sr}$ was measured by spectral synthesis of the two strongest resonance lines, Sr II 4077 and $4215 \AA$. Both of the lines are affected by HFS of the odd isotope ${ }^{87} \mathrm{Sr}$, and a fraction of 0.22 was adopted for this isotope for synthesis, according to Arlandini et al. (1999) for a pure $r$-process production of strontium. The correction of HFS and isotopic splitting has resulted in an increase of 0.15 dex for the abundance of $\mathrm{Sr}$, i.e., from -0.25 to -0.10 . For $\mathrm{Y}$ and $\mathrm{Zr}$, the abundances were derived from the EWs of three and two isolated and clear atomic lines, respectively. Abundances of these elements in LAMOST J110901.22+075441.8 are quite similar to other $r-$ II stars, 
and are higher than the average abundance ratio of "normal" EMP halo stars with similar metallicities (Fig. 2).

The second $r$-process peak elements. In the region of the second peak, abundances were determined for eight elements $\mathrm{Ba}, \mathrm{La}, \mathrm{Ce}, \mathrm{Pr}, \mathrm{Nd}, \mathrm{Sm}, \mathrm{Eu}$, and $\mathrm{Dy}$. Four isolated barium lines could be measured for LAMOST J110901.22+075441.8, including the Ba II $4554 \AA$ resonance line and the three subordinate lines, Ba II 5853, 6141, and $6497 \AA$. The three subordinate lines are almost free from HFS effects, and derive quite similar $\mathrm{Ba}$ abundances with a difference of about 0.01 dex. However, the resonance line is strongly affected by HFS. Even if we take the HFS splitting for spectral synthesis into account, the Ba II $4554 \AA$ line still results in a Ba abundance with 0.20 dex higher than that from the subordinate lines. We suspect that this difference is mainly caused by the NLTE effect (Mashonkina \& Christlieb 2014), which is not included in our analysis for this paper. Therefore, the adopted Ba abundance of LAMOST J110901.22+075441.8 was solely based on the three subordinate lines. HFS splitting was also accounted for La (Lawler et al. 2001a), which resulted in a difference of 0.3 dex in $\log \epsilon(\mathrm{La})$. Europium is another element for which HFS and isotope splitting has been accounted for. Eu II $4129 \AA$ is the only europium line detected in the spectrum, which consists of more than 30 components in total. The Eu abundance of LAMOST J110901.22+075441.8 was thus derived by synthesizing the Eu II $4129 \AA$ line, taking the HFS data from Lawler et al. (2001b) and the meteoritic isotopic abundance ratio of ${ }^{151} \mathrm{Eu}:{ }^{153} \mathrm{Eu}=47.8: 52.2$. As shown in Fig. 2, abundance ratios of the second peak elements relative to iron for LAMOST J110901.22+075441.8 are notably higher than normal EMP giants with similar metallicities.

\section{HEAVY-ELEMENT ABUNDANCE PATTERN OF LAMOST J110901.22+075441.8}

LAMOST J110901.22+075441.8 is the cool $r$-II giant with the lowest metallicity yet known. Fig. 3 compares its abundance pattern of heavy elements with those of two well-studied cool $r$-II giants with $T_{\text {eff }}<5000$ K. CS 22892-052 (Sneden et al. 2003), and CS 31082-001 (Hill et al. 2002). Abundances of the comparison stars are scaled to match the abundance of La in LAMOST J110901.22+075441.8. Previous studies have indicated that the pattern of the heavy-element abundances in these two comparison stars are tracing the main component of the $r$-process (Sneden et al. 2003; Roederer et al. 2014). It is clear from the comparison that in the range from Sr to Dy, the abundance pattern of the neutron-capture elements in LAMOST J110901.22+075441.8 is very similar to the two cool $r$-II giants, and thus the main $r$-process. For example, the dispersion of the average difference of $\log \epsilon(\mathrm{x})$ values between LAMOST J110901.22+075441.8 and the two comprison $r-$ II stars are about 0.19 dex (vs. CS 22892-052) and 0.15 dex (vs. CS 31082-001), respectively, which is comparable to the $1 \sigma$ error in our elemental abundance determinations. The similar chemical abundance pattern observed in neutron-capture elements suggest that there shall be a common origin of these elements in the classical $r$-process.

For comparison, the SSr patterns predicted by Bisterzo et al. (2014) and Arlandini et al. (1999) are also displayed in Fig. 3, respectively in solid and dashed lines. These two sets of solar $r$-components are very much similar except elements with significant contribution of $s$-process to their solar abundances, especially for the light trans-iron elements such as Sr, Y, and Zr. However, as discussed in Mashonkina \& Christlieb (2014), it would be difficult to firmly conclude on the relation of light trans-iron elements between $r$-II stars and the solar $r$-process, due to the large uncertainty in the solar $r$-residuals. When compared to the predicted abundances by Bisterzo et al. (2014) and those by Arlandini et al. (1999), the elements in the range from Ba through Dy in LAMOST J110901.22+075441.8 are found to match the scaled SSr pattern very well, with a dispersion of 0.13 dex and 0.16 dex about the average abundance differences of the eight second peak elements, respectively. The observed match is in line with previous studies on other $r$-process rich stars, and provides additional evidence of universal production ratio of these elements during the evolution of the Galaxy.

Previous studies on metal-poor halo stars suggest that there exist distinct mechanisms between the production of light trans-iron elements and that of heavier elements beyond Ba (Aoki et al. 2005; François et al. 2007; Mashonkina et al. 2007). By choosing Sr (Zr) and Eu to represent the first and 


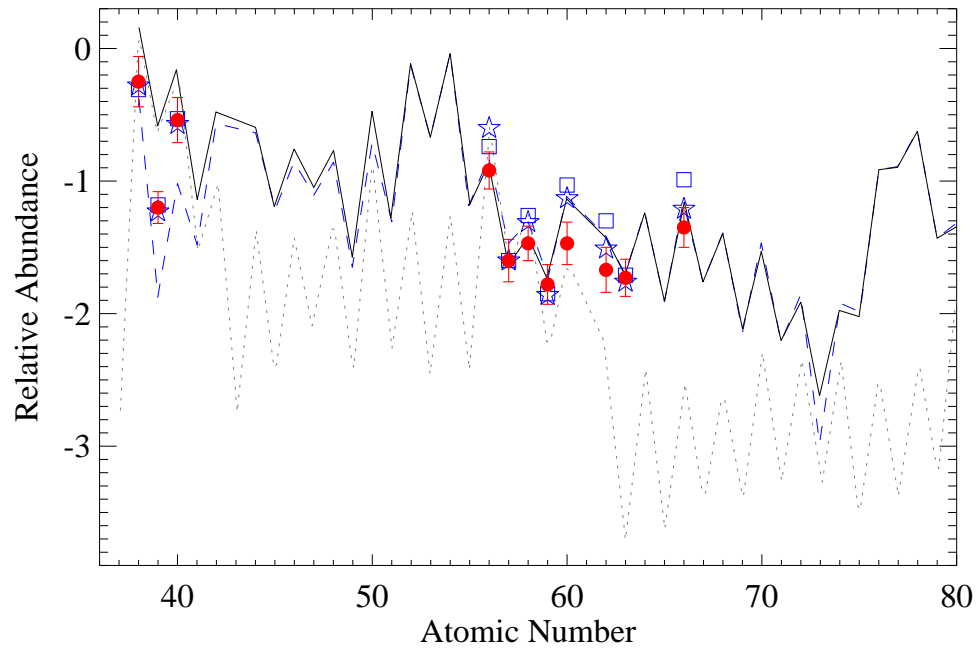

Fig. 3 Heavy-element abundance patterns of LAMOST J110901.22+075441.8 (filled circles) and r-II stars with $T_{\text {eff }}$ cooler than $5000 \mathrm{~K}$, including CS 22892-052 (Sneden et al. 2003, squares), and CS 31082-001 (Hill et al. 2002, stars). The element abundances are scaled to match La in LAMOST J110901.22+075441.8. The solid curve refers to the SSr abundance patterns from Bisterzo et al. (2014), and the dashed and dotted lines indicate those of the $r-$ and $s$-process components in the SSr from Arlandini et al. (1999), respectively.

second neutron-capture element peaks, we could inspect the $\mathrm{Sr} / \mathrm{Eu}$ and $\mathrm{Zr} / \mathrm{Eu}$ abundance ratios of the $r-\mathrm{II}$ stars. Fig. $4 \mathrm{a}$ and $4 \mathrm{~b}$ show the distribution of abundance ratios of $\log \mathrm{Sr} / \mathrm{Eu}$ and $\log \mathrm{Zr} / \mathrm{Eu}$ of known $r-$ II stars including LAMOST J110901.22+075441.8. The average value of $\log \mathrm{Sr} / \mathrm{Eu}$ and $\log \mathrm{Zr} / \mathrm{Eu}$ are $1.39 \pm 0.11$ and $1.32 \pm 0.12$ (dotted lines), which are consistent with the results of previous studies (e.g. Mashonkina et al. 2010), and are relatively smaller than those of normal EMP stars. Also, an average value of $\log \mathrm{Ba} / \mathrm{Eu}$ of $0.98 \pm 0.11$ (the dotted line in Fig. $4 \mathrm{c}$ ) is derived from the $12 r-$ II stars, which well agrees with the predicted value of 0.93 for the pure $r$-process production of heavy elements (Arlandini et al. 1999), which indicates that the environment from which these stars were formed only contained small amount of $s$-nuclei. The distribution of the abundance ratios as shown in Fig. 4 also suggests a common origin among the first and second $r$-process peak elements in strongly $r$-process enhanced stars.

\section{CONCLUSION}

LAMOST J110901.22+075441.8 has been selected as a candidate of EMP star from LAMOST spectroscopic survey, and followed-up obtaining a high-resolution spectrum with Subaru/HDS. The relatively high quality Subaru/HDS spectrum enables us to determine accurate parameters and elemental abundances for 23 species, including 11 elements in the nuclear charge range of $Z=38-66$ which covers the light trans-iron and the second $r$-process peak elements. Detailed abundance analysis confirms that LAMOST J110901.22+075441.8 is a strongly $r$-process enhanced EMP star having $[\mathrm{Eu} / \mathrm{Fe}]=+1.16$. It is also found that in the range from Sr through Dy, LAMOST J110901.22+075441.8 presents very similar abundance patterns of the elements to the well studied cool $r-$ II giants, CS 22892-052 and CS 31082-001, whose patterns of heavy elements can be well explained by a main component of the $r$-process. Therefore LAMOST J110901.22+075441.8 is a newly discovered member of the small 


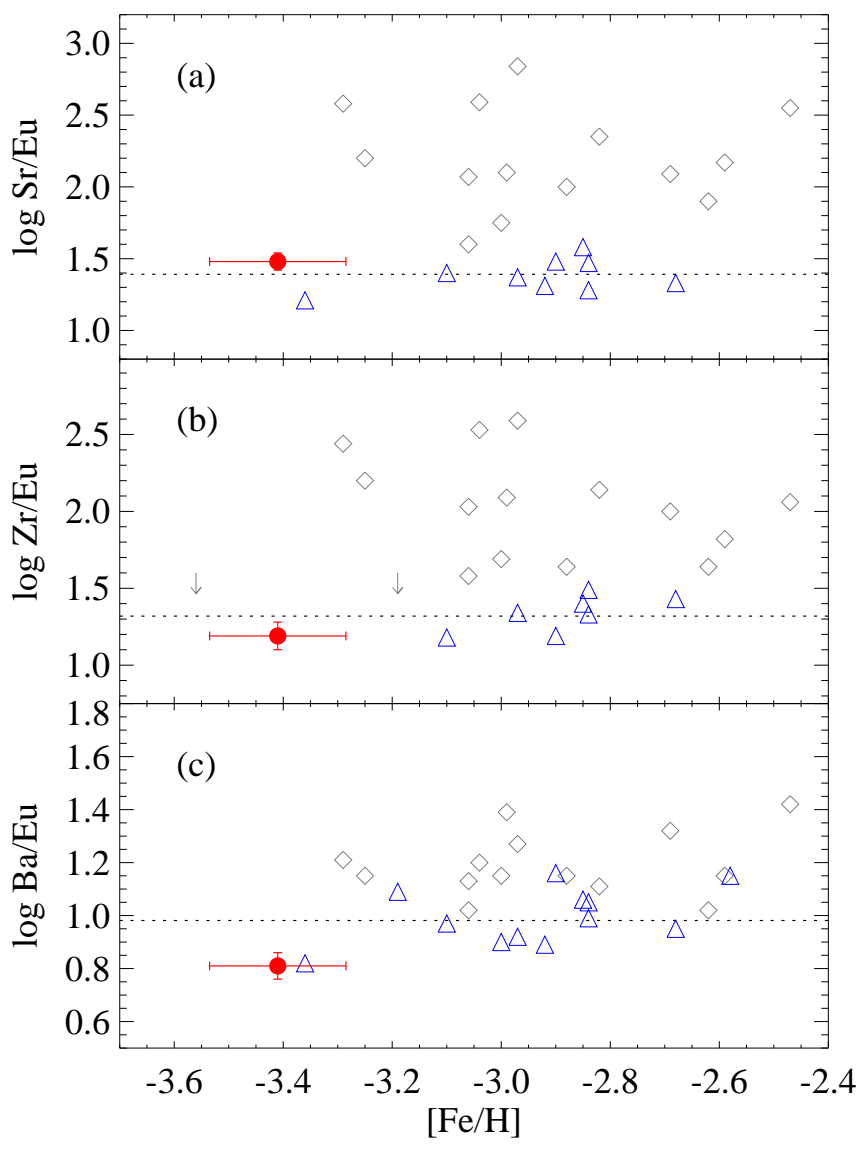

Fig. 4 The distribution of the $\log \mathrm{Sr} / \mathrm{Eu}(\mathrm{a}), \log \mathrm{Zr} / \mathrm{Eu}(\mathrm{b})$, and $\log \mathrm{Ba} / \mathrm{Eu}$ (c) abundance ratios in $r$-II stars (LAMOST J110901.22+075441.8 in filled circles, and literature $r$-II stars in triangles), and regular EMP giants from "First Stars" (François et al. 2007, diamonds for abundances and arrows for upper limits). The dotted lines indicate the average abundance ratio of the known $r$-II stars, as described in the text.

sample of currently known $r$-II stars, with the lowest metallicity of $[\mathrm{Fe} / \mathrm{H}] \sim-3.4$ among the $r-$ II giants.

The abundance pattern from Ba through Dy in LAMOST J110901.22+075441.8 can be well matched by the scaled Solar $r$-process pattern prediction by Arlandini et al. (1999) and Bisterzo et al. (2014). However, the large uncertainty of the Solar $r$-residuals for the first $r$-process peak elements leads to difficulties to draw any conclusion concerning the relation between the light trans-iron elements in LAMOST J110901.22+075441.8 (and other $r$-II stars) and the Solar $r$-process. 
Apart from different metallicities, LAMOST J110901.22+075441.8 presents quite similar abundance ratios of $\mathrm{Sr} / \mathrm{Eu}$ and $\mathrm{Zr} / \mathrm{Eu}$ compared with previously well studied $r-\mathrm{II}$ stars, and further confirms that LAMOST J110901.22+075441.8 belongs to the group of $r-$ II stars. LAMOST J110901.22+075441.8 turns to be the lowest metallicity $r$-II star with measured $\mathrm{Zr}$ abundance. The extreme enhancement in $[\mathrm{Eu} / \mathrm{Fe}]$ and low $\mathrm{Sr} / \mathrm{Eu}(\mathrm{Zr} / \mathrm{Eu}, \mathrm{Ba} / \mathrm{Eu}$ as well) in LAMOST J110901.22+075441.8 stars suggest a single or very few nucleosynthesis events as is the case for other $r-$ II stars.

However, due to limited wavelength coverage of the snapshot spectrum, our analysis does not include any elements heavier than Dy. To investigate abundance of the third $r$-process peak elements of LAMOST J110901.22+075441.8, we will further obtain spectra with higher quality and higher resolution which covers the UV band to investigate the chemical abundances of heavier elements beyond Dy.

Acknowledgements We are grateful to the anonymous referee for helping improve the paper. H.N.L. and G.Z. acknowledge supports by NSFC grants No. 11103030, 11233004, and 11390371. W.A. and T.S. are supported by the JSPS Grant-in-Aid for Scientific Research (S:23224004). S.H. is supported by the JSPS Grant-in-Aid for Scientific Research (c:26400231). N.C. acknowledges support from Sonderforschungsbereich 881 "The Milky Way System” (subproject A4) of the German Research Foundation (DFG). Guoshoujing Telescope (the Large Sky Area Multi-Object Fiber Spectroscopic Telescope, LAMOST) is a National Major Scientific Project built by the Chinese Academy of Sciences. Funding for the project has been provided by the National Development and Reform Commission. LAMOST is operated and managed by the National Astronomical Observatories, Chinese Academy of Sciences. This work is based on data collected at the Subaru Telescope, which is operated by the National Astronomical Observatory of Japan.

\section{References}

Alonso, A., Arribas, S., \& Martínez-Roger, C. 1999, A\&AS, 140, 2613

Alonso, A., Arribas, S., \& Martínez-Roger, C. 2001, A\&A, 376, 10393

Aoki, W., Beers, T. C., Christlieb, N., et al. 2007, ApJ, 655, 4926

Aoki, W., Beers, T. C., Honda, S., \& Carollo, D. 2010, ApJ, 723, L201 2, 4, 6

Aoki, W., Beers, T. C., Lee, Y. S., et al. 2013, AJ, 145, 13 2, 3

Aoki, W., Honda, S., Beers, T. C., et al. 2005, ApJ, 632, 611 3, 7

Arlandini, C., Käppeler, F., Wisshak, K., et al. 1999, ApJ, 525, 886 6, 7, 8, 9

Asplund, M., Grevesse, N., Sauval, A. J., \& Scott, P. 2009, ARA\&A, 47, 4814

Barklem, P. S., Christlieb, N., Beers, T. C., et al. 2005, A\&A, 439, 1296

Beers, T. C., \& Christlieb, N. 2005, ARA\&A, 43, 531 2, 6

Bisterzo, S., Travaglio, C., Gallino, R., Wiescher, M., \& Käppeler, F. 2014, ApJ, 787, 10 7, 8, 9

Burris, D. L., Pilachowski, C. A., Armandroff, T. E., et al. 2000, ApJ, 544, 3022

Castelli, F., \& Kurucz, R. L. 2003, in Modelling of Stellar Atmospheres, IAU Symposium, vol. 210, edited by N. Piskunov, W. W. Weiss, \& D. F. Gray, 20P-+ 4

Cayrel, R. 1988, in The Impact of Very High S/N Spectroscopy on Stellar Physics, IAU Symposium, vol. 132, edited by G. Cayrel de Strobel \& M. Spite, 3454

Cayrel, R., Depagne, E., Spite, M., et al. 2004, A\&A, 416, 1117 4, 5, 6

Christlieb, N., Beers, T. C., Barklem, P. S., et al. 2004, A\&A, 428, 10276

Cui, X.-Q., Zhao, Y.-H., Chu, Y.-Q., et al. 2012, Research in Astronomy and Astrophysics, 12,11972

François, P., Depagne, E., Hill, V., et al. 2007, A\&A, 476, 935 6, 7, 9

Frebel, A., Casey, A. R., Jacobson, H. R., \& Yu, Q. 2013, ApJ, 769, 573

Frebel, A., Christlieb, N., Norris, J. E., et al. 2007, ApJ, 660, L117 2

Goriely, S., Sida, J.-L., Lemaître, J.-F., et al. 2013, Physical Review Letters, 111, 2425022

Hayek, W., Wiesendahl, U., Christlieb, N., et al. 2009, A\&A, 504, 511 2, 6

Hill, V., Plez, B., Cayrel, R., et al. 2002, A\&A, 387, 560 2, 6, 7, 8 
Honda, S., Aoki, W., Kajino, T., et al. 2004, ApJ, 607, 474 2, 6

Lai, D. K., Bolte, M., Johnson, J. A., et al. 2008, ApJ, 681, 15246

Lawler, J. E., Bonvallet, G., \& Sneden, C. 2001a, ApJ, 556, 4527

Lawler, J. E., Wickliffe, M. E., den Hartog, E. A., \& Sneden, C. 2001b, ApJ, 563, 10757

Li, H.-N., Zhao, G., Christlieb, N., et al. 2015, ApJ, 798, 1102

Luo, A.-L., Zhang, H.-T., Zhao, Y.-H., et al. 2012, Research in Astronomy and Astrophysics, 12, 1243 2

Luo, A.-L., Zhao, Y.-H., Zhao, G., et al. 2015, ArXiv e-prints 2

Mashonkina, L., \& Christlieb, N. 2014, A\&A, 565, A123 7

Mashonkina, L., Christlieb, N., Barklem, P. S., et al. 2010, A\&A, 516, A46 2, 3, 6, 8

Mashonkina, L. I., Vinogradova, A. B., Ptitsyn, D. A., Khokhlova, V. S., \& Chernetsova, T. A. 2007, Astronomy Reports, 51, 9037

McWilliam, A. 1997, ARA\&A, 35, 503 5, 6

Roederer, I. U., Cowan, J. J., Preston, G. W., et al. 2014, MNRAS, 445, 29707

Sneden, C. 1973, ApJ, 184, 8394

Sneden, C., Cowan, J. J., \& Gallino, R. 2008, ARA\&A, 46, 241 2, 6

Sneden, C., Cowan, J. J., Lawler, J. E., et al. 2003, ApJ, 591, 936 2, 6, 7, 8

Sneden, C., McWilliam, A., Preston, G. W., et al. 1996, ApJ, 467, 8192

Sneden, C., Preston, G. W., McWilliam, A., \& Searle, L. 1994, ApJ, 431, L27 2

Sobeck, J. S., Kraft, R. P., Sneden, C., et al. 2011, AJ, 141, 1754

Spite, M., Cayrel, R., Plez, B., et al. 2005, A\&A, 430, 655 4, 6

Spite, M., \& Spite, F. 1978, A\&A, 67, 232

Woosley, S. E., Wilson, J. R., Mathews, G. J., Hoffman, R. D., \& Meyer, B. S. 1994, ApJ, 433, 2292

Zhao, G., Chen, Y., Shi, J., et al. 2006, Chinese Journal of Astronomy and Astrophysics, 6, 2652

Zhao, G., Zhao, Y.-H., Chu, Y.-Q., Jing, Y.-P., \& Deng, L.-C. 2012, Research in Astronomy and Astrophysics, 12, 7232 